\title{
A Deterministic Approach Used for Solving the Problem of Positioning and Angular Orientation Defining of Onboard Direction-Finder Antenna Based on the Results of Radio Direction Finding of Radio Reference Points
}

\author{
Valery N. Tyapkin and Alexander D. Vinogradov* \\ Siberian Federal University \\ Krasnoyarsk, Russian Federation
}

Received 24.06.2019, received in revised form 03.10.2019, accepted 21.01.2020

Abstract. The paper considers the possibilities and conditions for the unambiguous determination of the coordinates and angular orientation of the onboard direction - finder antenna placed on a moving object, based on the results of the azimuth-elevation radio direction finding of radio reference points. The coordinates of a moving object are determined by taken at one or several receiving points on board of a moving object measurements of radio signal delay time emitted simultaneously by at least three radio reference points. The unambiguous angular orientation in space is determined by measuring the viewing angles of at least three radio reference points of at least two direction finding pairs of receiving points with intersecting (non-collinear) bases. The article presents the mathematical features of determining the spatial position of onboard DF antenna, a general approach used for solving the problem of azimuth-elevation radio direction finding of three radio reference points, methods for determining the distance to radio reference points based on the results of their azimuth-elevation radio direction finding, and the analysis of the research results.

Keywords: azimuth-angle radio direction finding, radio reference point, onboard direction-finder antenna, navigation system receiver, source of radio emission.

Citation: Tyapkin V.N., Vinogradov A.D. A deterministic approach used for solving the problem of positioning and angular orientation defining of onboard direction-finder antenna based on the results of radio direction finding of radio reference points, J. Sib. Fed. Univ. Eng. \& Technol., 2020, 13(3), 289-310. DOI: 10.17516/1999-494X-0222

(c) Siberian Federal University. All rights reserved

This work is licensed under a Creative Commons Attribution-Non Commercial 4.0 International License (CC BY-NC 4.0).

* Corresponding author E-mail address: a-glonass@yandex.ru 


\title{
Детерминированный подход к решению задачи
}

\section{определения координат и угловой ориентации}

\section{бортовой пеленгаторной антенны}

\section{по результатам радиопеленгования радиоориентиров}

\author{
В.Н. Тяпкин, А.Д. Виноградов \\ Сибирский федеральный университет \\ Российская Федераџия, Красноярск
}

\begin{abstract}
Аннотация. В работе рассматривается исследование возможности и условий однозначного определения координат и угловой ориентации бортовой пеленгаторной антенны (БПА), размещенной на подвижном объекте, по результатам азимутально-угломестного радиопеленгования радиоориентиров. Координаты подвижного объекта определяют по результатам измерений в одной или нескольких точках приема на борту подвижного объекта времен задержки радиосигналов, синхронно излучаемых не менее чем тремя радиоориентирами, а однозначная угловая ориентация в пространстве - путем измерения углов визирования не менее трех радиоориентиров не менее чем двумя пеленгационными парами точек приема с пересекающимися (неколлинеарными) базами. В статье приведены математические особенности определения пространственного положения БПА, общий подход к решению задачи при азимутально-угломестном радиопеленговании трех радиоориентиров, способы определения дальности до радиоориентиров по результатам их азимутально-угломестного радиопеленгования и анализ результатов исследования.
\end{abstract}

Ключевые слова: азимутально-угломестный радиопеленг, радиоориентир, бортовая пеленгаторная антенна, навигационный приемник, источник радиоизлучения.

Цитирование: Тяпкин, В.Н. Детерминированный подход к решению задачи определения координат и угловой ориентации бортовой пеленгаторной антенны по результатам радиопеленгования радиоориентиров / В.Н. Тяпкин, А.Д. Виноградов // Журн. Сиб. федер. ун-та. Техника и технологии, 2020. 13(3). С. 289-310. DOI: 10.17516/1999-494X0222

\section{Введение}

Для определения координат и угловой ориентации подвижных объектов воздушного, морского или наземного базирования в настоящее время используют спутниковые радионавигационные системы $[1,2]$, основанные на излучении радиосигналов реперными источниками радиоизлучения (ИРИ), размещенными на спутниках с известными координатами, называемые в общем случае радиоориентирами или радионавигационными точками [3], и приеме этих радиосигналов в нескольких пространственно разнесенных точках, размещенных на подвижном объекте, выполняющих функции бортовой пеленгаторной антенны (БПА) и обеспечивающих реализацию фазового метода радиопеленгования $[2,4,5]$. При этом координаты подвижного объекта определяют по результатам измерений в одной или нескольких точках приема на борту подвижного объекта времен задержки радиосигналов, синхронно излучаемых не менее чем тремя радиоориентирами, а однозначная угловая ориентация в пространстве - путем измерения углов визирования не менее трех радиоориентиров не менее чем двумя пеленгационными парами точек приема с пересекающимися (неколлинеарными) базами. Следует отметить, что угол визирования представляет собой угол между отрезком линии, проходящей через пелен- 
гационную пару точек приема, и направлением на радиоориентир, проходящим через одну из точек приема пеленгационной пары, координаты которой определены вышеупомянутым образом $[2,4,5]$. То есть координаты и угловая ориентация подвижных объектов определяются спутниковыми радионавигационными системами дальномерно-угломерным методом, реализуемым при условии синхронного излучения радиосигналов радиоориентиров.

В известных работах по радионавигации [6-10] исследованы различные способы определения координат в пространстве подвижного объекта угломерным методом путем радиопеленгования с борта подвижного объекта, оснащенного бортовыми автономными навигационными датчиками и системами (инерциальными, геомагнитными) [11-14], радиоориентиром, реализуемым без предъявления требований к синхронности излучения радиосигналов радиоориентирами. Однако возможности одновременного и однозначного определения координат и угловой ориентации в пространстве подвижного объекта путем азимутально-угломестного радиопеленгования (определения азимута и угла места источников радиоизлучения) с борта подвижного объекта радиоориентиров без использования вспомогательной информации от автономных навигационных датчиков и систем в известных работах по радионавигации [1-14] не исследованы. При этом возникает необходимость решения задачи определения условий однозначности определения координат и угловой ориентации в пространстве подвижного объекта, оснащенного БПА, определяющих минимально возможное число и ограничения на взаимное пространственное расположение БПА и радиоориентиров.

Цель работы - исследование возможности и условий однозначного определения координат и угловой ориентации бортовой пеленгаторной антенны, размещенной на подвижном объекте, по результатам азимутально-угломестного радиопеленгования радиоориентиров.

Постановка задачи. Будем считать, что $N$ радиоориентиров размещены в $i$-х точках $M_{i}$ пространства (где $i=1,2, \ldots, N)$ с известными координатами $M_{i}\left(x_{i}, y_{i}, z_{i}\right)$ в нормальной земной системе координат (НЗСК) $\Sigma_{\text {нз }}=\{O, X, Y, Z\}$, представляющей собой левую пространственную прямоугольную декартовую систему координат, начало $O$ которой фиксировано по отношению к Земле, ось абсцисс $O X$ которой, находящаяся в горизонтальной плоскости, совпадает с северным направлением истинного или магнитного меридиана или вертикальной линии координатной сетки плоской прямоугольной геодезической системы координат, ось аппликат $O Z$ которой перпендикулярна горизонтальной плоскости и направлена вверх по вертикали, а ось ординат $O Y$ которой, находящаяся в горизонтальной плоскости $X O Y$, дополняет систему до левой пространственной прямоугольной декартовой системы координат [15]. Отсчет углов (азимута $\alpha$ и угла места $\varepsilon$ ) при азимутально-угломестном радиопеленговании реперных ИРИ с борта подвижного объекта, на котором размещена БПА, осуществляется в связанной системе координат БПА $\Sigma_{\text {св }}=\left\{O^{\prime}, X^{\prime}, Y^{\prime}, Z^{\prime}\right\}$, представляющей собой левую пространственную прямоугольную декартовую систему координат, начало $O^{\prime}$ которой помещено в фазовом центре (ФЦ) БПА, осями которой служат продольная, поперечная и нормальная осевые линии БПА, являющиеся осями абсцисс $O^{\prime} X^{\prime}$, ординат $O^{\prime} Y^{\prime}$ и аппликат $O^{\prime} Z^{\prime}$ соответственно [16]. При этом азимут $\alpha$ представляет собой угол между проекцией направления от ФЦ БПА на радиоориентир на азимутальную плоскость $O^{\prime} X^{\prime} Y^{\prime}$, содержащую продольную и поперечную осевые линии БПА, и положительным направлением продольной осевой линии $O^{\prime} X^{\prime}$ БПА, измеряемый в градусах в пределах от $0^{\circ}$ до $360^{\circ}$ и отсчитываемый от положительного направления продольной осевой

$$
-291-
$$


линии $O^{\prime} X^{\prime}$ БПА по ходу часовой стрелки, если смотреть в направлении навстречу положительному направлению нормальной осевой линии $O^{\prime} Z^{\prime}$ БПА. Угол места $\varepsilon$ есть угол между направлением от ФЦ БПА на радиоориентир и проекцией вышеупомянутого направления на азимутальную плоскость $O^{\prime} X^{\prime} Y^{\prime}$, содержащую продольную и поперечную осевые линии БПА, измеряемый в градусах в пределах от $0^{\circ}$ до $\pm 90^{\circ}$, отсчитываемый от вышеупомянутой проекции на азимутальную плоскость $O^{\prime} X^{\prime} Y^{\prime}$ с положительным или отрицательным знаками в случае, если радиоориентир находится соответственно выше или ниже азимутальной плоскости $O^{\prime} X^{\prime} Y^{\prime}$, проходящей через ФЦ БПА.

Пространственное положение и угловую ориентацию БПА в НЗСК $\Sigma_{\text {нз }}=\{O, X, Y, Z\}$ будем характеризовать: во-первых, координатами $x, y$ и $z$ точки $M_{0}(x, y, z)$ размещения ФЦ $O^{\prime}$ БПА в НЗСК $\Sigma_{\text {нз }}=\{O, X, Y, Z\}$; во-вторых, тремя углами Эйлера [17]: углами курса $\psi$, тангажа $\mu$ и крена $\vartheta$, определяющими угловую ориентацию продольной $O^{\prime} X^{\prime}$, поперечной $O^{\prime} Y^{\prime}$ и нормальной $O^{\prime} Z^{\prime}$ осевых линий БПА в нормальной земной подвижной системе координат (НЗПСК) $\Sigma_{\text {нзп }}=\left\{O^{\prime}, X, Y, Z\right\}$, представляющей собой левую пространственную прямоугольную декартовую систему координат, начало $O^{\prime}$ которой помещено в ФЦ $O^{\prime}$ БПА, а оси абсцисс $O^{\prime} X$, ординат $O^{\prime} Y$ и аппликат $O^{\prime} Z$ которой направлены так же, как и оси соответственно абсцисс $O X$, ординат $O Y$ и аппликат $O Z$ НЗСК $\Sigma_{\text {нз }}=\{O, X, Y, Z\}$ [17-19]. При этом угол курса $\psi$ БПА представляет собой угол между проекцией на горизонтальную плоскость $O X^{\prime} Y$ НЗПСК $\Sigma_{\text {нзп }}=\left\{O^{\prime}, X, Y, Z\right\}$ положительного направления продольной осевой линии $O^{\prime} X^{\prime}$ БПА и линией $O^{\prime} X$, расположенной в горизонтальной плоскости $O X^{\prime} Y$, проходящей через ФЦ $O^{\prime}$ БПА, принятой за начало отсчета, измеряемый в градусах в пределах от $0^{\circ}$ до $360^{\circ}$ и отсчитываемый от вышеупомянутой линии $O^{\prime} X$ по ходу часовой стрелки, если смотреть в направлении вниз по вертикали, проходящей через ФЦ $O^{\prime}$ БПА. Угол тангажа $\mu$ БПА есть угол между продольной осевой линией $O^{\prime} X^{\prime}$ БПА и горизонтальной плоскостью $O X^{\prime} Y$ НЗПСК $\Sigma_{\text {нзп }}=\left\{O^{\prime}, X, Y, Z\right\}$, проходящей через ФЦ $O^{\prime}$ БПА, измеряемый в градусах в пределах от $0^{\circ}$ до $\pm 90^{\circ}$, отсчитываемый от горизонтальной плоскости $O X^{\prime} Y$ с положительным или отрицательным знаками в случае, если положительное направление продольной осевой линии $O^{\prime} X^{\prime}$ БПА находится соответственно выше или ниже горизонтальной плоскости $O X^{\prime} Y$. Угол крена $\vartheta$ БПА представляет собой угол между поперечной осевой линией $O^{\prime} Y^{\prime}$ БПА и осью ординат $O^{\prime} Y_{\psi}$ смещенной НЗПСК $\Sigma_{\text {нзпч }}=\left\{O^{\prime}, X_{\psi}, Y_{\psi}, Z\right\}$, представляющей собой НЗПСК, оси абсцисс $O^{\prime} X_{\psi}$ и ординат $O^{\prime} Y_{\psi}$ которой смещены относительно осей соответственно абсцисс $O^{\prime} X$ и ординат $O^{\prime} Y$ НЗПСК $\Sigma_{\text {нзп }}=\left\{O^{\prime}, X, Y, Z\right\}$ в положение, при котором угол курса $\psi$ БПА равен нулю. Угол крена $\vartheta$ БПА измеряется в градусах в пределах от $0^{\circ}$ до $\pm 90^{\circ}$ и отсчитывается от оси ординат $O^{\prime} Y_{\psi}$ смещенной НЗПСК $\Sigma_{\text {нзп }}=\left\{O^{\prime}, X_{\psi}, Y_{\psi}, Z\right\}$ с положительным или отрицательным знаками в случае, если положительное направление оси ординат $O^{\prime} Y_{\psi}$ смещенной НЗПСК $\Sigma_{\text {нзпш }}=\left\{O^{\prime}, X_{\psi}, Y_{\psi}, Z\right\}$ совмещается с положительным направлением поперечной осевой линии $O^{\prime} Y^{\prime}$ БПА поворотом вокруг продольной осевой линии $O^{\prime} X^{\prime}$ БПА соответственно по ходу или против хода часовой стрелки, если смотреть в положительном направлении продольной осевой линии $O^{\prime} X^{\prime}$ БПА.

При одновременном азимутально-угломестном радиопеленговании с борта подвижного объекта $N$ радиоориентиров, размещенных в $i$-х точках пространства с известными координатами $M_{i}\left(x_{i}, y_{i}, z_{i}\right)$, можно получить соответствующую совокупность $i$-х пар азимутов $\alpha_{i}$ и углов места $\varepsilon_{i}($ где $i=1,2, \ldots N)$. Так как пространственное положение и угловая ориентация БПА опре- 
деляются шестью неизвестными параметрами (тремя координатами $x, y$ и $z$ и тремя углами $\psi$, $\mu$ и $)$, то для их однозначного определения необходимо измерять не менее шести параметров, какими в рамках рассматриваемой задачи являются не менее трех пар азимутов $\alpha_{i}$ и углов места $\varepsilon_{i}$, получаемых, соответственно, в результате радиопеленгования радиоориентиров с их общим числом $N \geq 3$. Детерминированный подход к решению задачи определения координат ФЦ БПА $x, y, z$ и углов $\psi, \mu$ и $\vartheta$, определяющих угловую ориентацию БПА, по результатам измерений не менее трех пар азимутов $\alpha_{i}$ и углов места $\varepsilon_{i}$ при азимутально-угломестном радиопеленговании не менее трех радиоориентиров можно представить в виде четырехэтапной процедуры, этапы которой предназначены для решения следующих частных задач:

- нахождение совокупности расстояний от ФЦ БПА до радиоориентиров;

- определение областей пространства, в которых совокупность расстояний от ФЦ БПА до радиоориентиров однозначна;

- определение координат ФЦ БПА;

- нахождение матрицы вращения и связанных с нею углов Эйлера, определяющих угловую ориентацию в пространстве БПА.

Система уравнений связи между неизвестной совокупностью расстояний от ФЦ БПА до радиоориентиров и соответствующей совокупностью известных (измеряемых) угловых параметров, возникающая при решении первой частной задачи на первом этапе, является нелинейной. Поэтому при азимутально-угломестном радиопеленговании с борта подвижного объекта трех радиоориентиров в общем случае произвольного взаимного пространственного расположения БПА относительно радиоориентиров решение вышеупомянутой определенной системы уравнений относительно совокупности расстояний от ФЦ БПА до трех радиоориентиров неоднозначно и может включать от одного до четырех решений. При увеличении размерности системы уравнений путем азимутально-угломестного радиопеленгования более чем трех радиоориентиров система уравнений связи становится переопределенной, и, соответственно, в случае учета погрешностей измерений азимутов и углов места она перестает быть совместной. Применение для ее решения стандартного метода наименьших квадратов приводит к повышению степени уравнений и, соответственно, к существенному усложнению процедуры определения искомых параметров. Поэтому в данной работе рассматриваем два случая, когда возможен детерминированный подход к решению первой и второй частных задач нахождения однозначной совокупности расстояний от ФЦ БПА до радиоориентиров: а) в случае азимутально-угломестного радиопеленгования трех радиоориентиров, не находящихся на одной прямой линии, когда система уравнений связи между искомыми и измеряемыми параметрами является определенной; б) в случае азимутально-угломестного радиопеленгования четырех радиоориентиров, три из которых расположены на одной прямой линии, когда система уравнений связи между искомыми и измеряемыми параметрами является переопределенной и совместной. Задачи третьего и четвертого этапов стандартны для радионавигации подвижных объектов [6-10], поэтому для их решения не требуется разрабатывать специальных методов. Ключевыми оказываются именно первые два этапа решения вышеупомянутых первой и второй частных задач. Структура возможных решений соответствующих нелинейных систем уравнений связи между искомыми и измеряемыми параметрами оказывается сложной, включая большое число вырожденных случаев. Без понимания данных особенностей затруднительно строить эффек- 
тивные вычислительные алгоритмы однозначного определения координат и угловой ориентации БПА, размещенной на подвижном объекте, по результатам азимутально-угломестного радиопеленгования радиоориентиров.

Общий подход к решению задачи при азимутально-угломестном радиопеленговании трех радиоориентиров. Если три радиоориентира размещены в точках $M_{1}\left(x_{1}, y_{1}, z_{1}\right), M_{2}\left(x_{2}, y_{2}, z_{2}\right)$ и $M_{3}\left(x_{3}, y_{3}, z_{3}\right)$ с заданными известными координатами, находящихся не на одной прямой линии, а ФЦ $O^{\prime}$ БПА, размещенной на подвижном объекте, - в точке $M_{0}$ с неизвестными координатами $M_{0}(x, y, z)$, то в общем случае указанные четыре точки в трехмерном пространстве образуют треугольную пирамиду, схематическое представление которой приведено на рис. 1 , где кроме вышеупомянутых параметров обозначены: $\ell_{i}$ - длина $i$-го бокового ребра $M_{0} M_{i}$ треугольной пирамиды $M_{0} M_{1} M_{2} M_{3} ; d_{i j}$ - длина ребра $M_{i} M_{j}$ основания $M_{1} M_{2} M_{3}$ треугольной пирамиды $M_{0} M_{1} M_{2} M_{3} ; \alpha_{i j}=\angle M_{i} M_{0} M_{j}$ - плоский угол при вершине $M_{0}$ между боковыми ребрами $M_{0} M_{i}$ и $M_{0} M_{j}$ треугольной пирамиды $M_{0} M_{1} M_{2} M_{3} ; i=1,2,3 ; j=1,2,3 ; i<j$. Пространственное положение точки $M_{0}$ размещения ФЦ $O^{\prime}$ БПА и точек $M_{i}$ размещения $i$-х радиоориентиров в НЗСК $\Sigma_{\text {нз }}=\{O, X, Y, Z\}$ будем также характеризовать радиус-векторами $\mathbf{r}_{0}=\left(x_{0}, y_{0}, z_{0}\right)$ и $\mathbf{r}_{i}=\left(x_{i}, y_{i}, z_{i}\right)$ соответственно, где $i=1,2,3$.

Допустим, что в результате одновременного азимутально-угломестного радиопеленгования $i$-х радиоориентиров с использованием БПА определены три пары азимутов $\alpha_{i}$ и углов места $\varepsilon_{i}$. Тогда в связанной системе координат БПА $\Sigma_{\text {св }}=\left\{O^{\prime}, X^{\prime}, Y^{\prime}, Z^{\prime}\right\}$ можно определить три единичных вектора $\mathbf{s}_{\text {св } i}$ направлений на $i$-е радиоориентиры в соответствии с соотношением

$$
\mathbf{s}_{\mathrm{cB} i}=\left(\cos \alpha_{i} \cos \varepsilon_{i}, \sin \alpha_{i} \cos \varepsilon_{i}, \sin \varepsilon_{i}\right),
$$

где $i=1,2,3$.

С учетом (1) косинусы плоских углов $\cos \alpha_{i j}$ при вершине $M_{0}$ треугольной пирамиды $M_{0} M_{1} M_{2} M_{3}$ (см. рис. 1) можно определить в соответствии с соотношением

$$
\cos \alpha_{i j}=\left(\mathbf{s}_{\mathrm{cB} i}, \mathbf{s}_{\mathrm{cB} j}\right)=\cos \varepsilon_{i} \cos \varepsilon_{j} \cos \left(\alpha_{i}-\alpha_{j}\right)+\sin \varepsilon_{i} \sin \varepsilon_{j},
$$

где $1 \leq i<j \leq 3$.

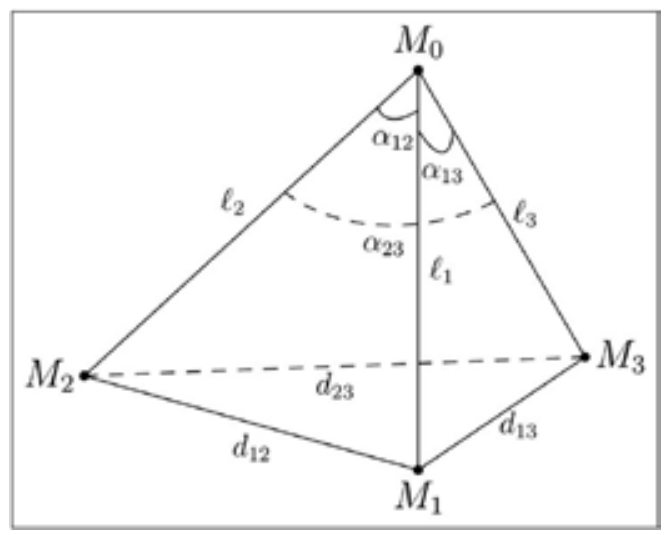

Рис. 1. Схема размещения в пространстве трех радиоориентиров и фазового центра БПА

Fig. 1. Spatial layout of three radio reference points and the onboard DF antenna phase center 
Следует отметить, что длины $d_{i j}$ ребер $M_{i} M_{j}$ основания $M_{1} M_{2} M_{3}$ треугольной пирамиды $M_{0} M_{1} M_{2} M_{3}$ (см. рис. 1) являются априорно известными параметрами и определяются в соответствии с соотношением

$$
d_{i j}=\sqrt{\left(x_{i}-x_{j}\right)^{2}+\left(y_{i}-y_{j}\right)^{2}+\left(z_{i}-z_{j}\right)^{2}}
$$

где $1 \leq i<j \leq 3$.

С учетом известных значений вышеупомянутых параметров $\cos \alpha_{12}, \cos \alpha_{13}, \cos \alpha_{23}, d_{12}, d_{13}$ и $d_{23}$ для определения трех неизвестных значений длин $\ell_{1}, \ell_{2}$ и $\ell_{3}$ боковых ребер треугольной пирамиды $M_{0} M_{1} M_{2} M_{3}$ (см. рис. 1) получаем следующую систему из трех уравнений:

$$
\left\{\begin{array}{l}
\ell_{1}^{2}+\ell_{2}^{2}-2 \ell_{1} \ell_{2} \cos \alpha_{12}=d_{12}^{2} \\
\ell_{1}^{2}+\ell_{3}^{2}-2 \ell_{1} \ell_{3} \cos \alpha_{13}=d_{13}^{2} \\
\ell_{2}^{2}+\ell_{3}^{2}-2 \ell_{2} \ell_{3} \cos \alpha_{23}=d_{23}^{2}
\end{array} .\right.
$$

Как показали вычислительные эксперименты, система уравнений (4) относительно искомых значений параметров $\ell_{1}, \ell_{2}$ и $\ell_{3}$ может иметь от одного до четырех решений в каждой из двух областей пространства, находящихся симметрично относительно плоскости расположения трех радиоориентиров. Структура этих решений и правила выбора правильного (однозначного) решения будут описаны далее. Предположим, что однозначные значения параметров $\ell_{1}, \ell_{2}, \ell_{3}$ из системы уравнений (4) определены. Для неизвестных координат $x, y$ и $z$ точки $M_{0}(x, y, z)$ расположения ФЦ БПА получаем следующую систему из трех уравнений:

$$
\left\{\begin{array}{l}
\left(x-x_{1}\right)^{2}+\left(y-y_{1}\right)^{2}+\left(z-z_{1}\right)^{2}=\ell_{1}^{2} \\
\left(x-x_{2}\right)^{2}+\left(y-y_{2}\right)^{2}+\left(z-z_{2}\right)^{2}=\ell_{2}^{2} \\
\left(x-x_{3}\right)^{2}+\left(y-y_{3}\right)^{2}+\left(z-z_{3}\right)^{2}=\ell_{3}^{2} .
\end{array}\right.
$$

Для решения системы уравнений (5) вычтем из второго и третьего уравнений первое уравнение и перенесем параметры, связанные с неизвестным значением $z$, в правые части уравнений, в результате чего относительно неизвестных значений координат $x$ и $y$ получаем следующую систему из двух уравнений:

$$
\left\{\begin{array}{l}
2 x\left(x_{1}-x_{2}\right)+2 y\left(y_{1}-y_{2}\right)=\ell_{2}^{2}-\ell_{1}^{2}+x_{1}^{2}-x_{2}^{2}+y_{1}^{2}-y_{2}^{2}+z_{1}^{2}-z_{2}^{2}-2 z\left(z_{1}-z_{2}\right) ; \\
2 x\left(x_{1}-x_{3}\right)+2 y\left(y_{1}-y_{3}\right)=\ell_{3}^{2}-\ell_{1}^{2}+x_{1}^{2}-x_{3}^{2}+y_{1}^{2}-y_{3}^{2}+z_{1}^{2}-z_{3}^{2}-2 z\left(z_{1}-z_{3}\right) .
\end{array}\right.
$$

Согласно правилу Крамера решение системы уравнений (6) относительно координат $x$ и $y$, зависящих от неизвестного значения координаты $z$, определяется соотношениями

$$
x=V^{-1}\left|\begin{array}{ll}
A(z) & 2\left(y_{1}-y_{2}\right) \\
B(z) & 2\left(y_{1}-y_{3}\right)
\end{array}\right| y=V^{-1}\left|\begin{array}{ll}
2\left(x_{1}-x_{2}\right) & A(z) \\
2\left(x_{1}-x_{3}\right) & B(z)
\end{array}\right|,
$$

где

$$
\begin{gathered}
A(z)=\ell_{2}^{2}-\ell_{1}^{2}+x_{1}^{2}-x_{2}^{2}+y_{1}^{2}-y_{2}^{2}+z_{1}^{2}-z_{2}^{2}-2 z\left(z_{1}-z_{2}\right) \\
B(z)=\ell_{3}^{2}-\ell_{1}^{2}+x_{1}^{2}-x_{3}^{2}+y_{1}^{2}-y_{3}^{2}+z_{1}^{2}-z_{3}^{2}-2 z\left(z_{1}-z_{3}\right) \\
-295-
\end{gathered}
$$


$V$ - определитель системы уравнений (6), определяемый соотношением

$$
V=2\left|\begin{array}{ll}
\left(x_{1}-x_{2}\right) & \left(y_{1}-y_{2}\right) \\
\left(x_{1}-x_{3}\right) & \left(y_{1}-y_{3}\right)
\end{array}\right| .
$$

Для однозначной разрешимости системы уравнений (6) в соответствии с (7) требуется, чтобы определитель $V$ системы уравнений (6) не был равен нулю, что с учетом (8) равносильно требованию того, чтобы точки $M_{1}\left(x_{1}, y_{1}, z_{1}\right), M_{2}\left(x_{2}, y_{2}, z_{2}\right)$ и $M_{3}\left(x_{3}, y_{3}, z_{3}\right)$ размещения радиоориентиров не находились на одной прямой, что напрямую вытекает из постановки задачи (см. рис. 1).

После подстановки соотношений (7) в одно из уравнений (5) получим квадратное уравнение относительно $z$. Полученное в результате решения вышеупомянутого квадратного уравнения значение координаты $z$ точки $M_{0}(x, y, z)$ расположения ФЦ БПА используется в качестве известного параметра при решении системы их двух уравнений (6) в соответствии с соотношениями (7) относительно значений координат $x$ и $y$ точки $M_{0}(x, y, z)$ расположения ФЦ БПА.

После нахождения координат точки $M_{0}(x, y, z)$ расположения ФЦ $O^{\prime}$ БПА и вычисления в НЗСК $\Sigma_{\text {нз }}=\{O, X, Y, Z\}$ радиус-векторов $\mathbf{r}_{0}=\left(x_{0}, y_{0}, z_{0}\right)$ и $\mathbf{r}_{i}=\left(x_{i}, y_{i}, z_{i}\right)$ можно определить три единичных вектора $\mathbf{s}_{\text {нзпi }}$ направлений на $i$-е радиоориентиры относительно ФЦ $O^{\prime}$ БПА в НЗПСК $\Sigma_{\text {нзп }}=\left\{O^{\prime}, X, Y, Z\right\}$ в соответствии с соотношением

$$
\mathbf{S}_{\text {нзп } i}=\left(s_{\text {нзпі } x}, s_{\text {нзпі } y}, s_{\text {нзп } i z}\right)=\frac{\mathbf{r}_{0}-\mathbf{r}_{i}}{\left|\mathbf{r}_{0}-\mathbf{r}_{i}\right|},
$$

где $\mathbf{s}_{\text {нзпі }}, \mathbf{s}_{\text {нзпіу }}$ и $\mathbf{s}_{\text {нзпіz }}-$ координаты $i$-го единичного вектора $\mathbf{s}_{\text {нзпі }}$ в НЗПСК $\Sigma_{\text {нзп }}=\left\{O^{\prime}, X, Y, Z\right\}$; $i=1,2,3$.

Определим квадратную матрицу $\mathbf{S}_{\text {нзп }}$ размера $3 \times 3$ координат трех полученных по формуле (9) единичных векторов $\mathbf{s}_{\text {нзп1 }}, \mathbf{s}_{\text {нзп2 }}$ и $\mathbf{s}_{\text {нзп3 }}$, записанных в столбцы, в соответствии с соотношением

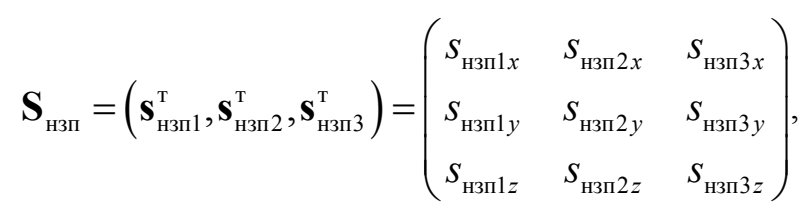

где $(\cdot)^{\mathrm{T}}-$ знак транспонирования.

По аналогии с (10) определим квадратную матрицу $\mathbf{S}_{\text {св }}$ размера $3 \times 3$ координат трех полученных по формуле (1) единичных векторов $\mathbf{s}_{\mathrm{cв} 1}, \mathbf{s}_{\mathrm{cв} 2}$ и $\mathbf{s}_{\mathrm{cв} 3}$ в связанной системе координат $\Sigma_{\text {св }}=\left\{O^{\prime}, X^{\prime}, Y^{\prime}, Z^{\prime}\right\}$, записанных в столбцы, в соответствии с соотношением

$$
\mathbf{S}_{\mathrm{cB}}=\left(\mathbf{s}_{\mathrm{cB} 1}^{\mathrm{T}}, \mathbf{s}_{\mathrm{cB} 2}^{\mathrm{T}}, \mathbf{s}_{\mathrm{cB} 3}^{\mathrm{T}}\right)=\left(\begin{array}{ccc}
\cos \alpha_{1} \cos \varepsilon_{1} & \cos \alpha_{2} \cos \varepsilon_{2} & \cos \alpha_{3} \cos \varepsilon_{3} \\
\sin \alpha_{1} \cos \varepsilon_{1} & \sin \alpha_{2} \cos \varepsilon_{2} & \sin \alpha_{3} \cos \varepsilon_{3} \\
\sin \varepsilon_{1} & \sin \varepsilon_{2} & \sin \varepsilon_{3}
\end{array}\right) .
$$

Квадратные матрицы $\mathbf{S}_{\text {нзп }}$ и $\mathbf{S}_{\text {св }}$ размеров $3 \times 3$ связаны между собой матричным соотношением

$$
\mathbf{S}_{\text {нзп }}=\xi_{\mathrm{cв}}^{\mathrm{н3п}} \mathbf{S}_{\text {св }},
$$


где $\xi_{\text {св }}^{\text {нз }}-$ квадратная матрица вращения размера $3 \times 3$ при переходе от связанной системы координат $\Sigma_{\mathrm{cв}}=\left\{O^{\prime}, X^{\prime}, Y^{\prime}, Z^{\prime}\right\}$ к НЗПСК $\Sigma_{\text {нзп }}=\left\{O^{\prime}, X, Y, Z\right\}$, определяемая соотношением

$$
\xi_{\mathrm{cB}}^{\mathrm{H3 \Pi}}=\left(\begin{array}{lll}
\xi_{X^{\prime} X} & \xi_{Y^{\prime} X} & \xi_{Z^{\prime} X} \\
\xi_{X^{\prime} Y} & \xi_{Y^{\prime} Y} & \xi_{Z^{\prime} Y} \\
\xi_{X^{\prime} Z} & \xi_{Y^{\prime} Z} & \xi_{Z^{\prime} Z}
\end{array}\right),
$$

где $\xi_{X^{\prime} X}, \xi_{X^{\prime} Y}, \xi_{X^{\prime} Z}, \xi_{Y^{\prime} X}, \xi_{Y^{\prime} Y}, \xi_{Y^{\prime} Z}, \xi_{Z^{\prime} X}, \xi_{Z^{\prime} Y}$ и $\xi_{Z^{\prime} Z}-$ направляющие косинусы осей связанной системы координат $\Sigma_{\text {св }}=\left\{O^{\prime}, X^{\prime}, Y^{\prime}, Z^{\prime}\right\}$ в НЗПСК $\Sigma_{\text {нзп }}=\left\{O^{\prime}, X, Y, Z\right\}$, связанные со значениями углов курса $\psi$, тангажа $\mu$ и крена $\vartheta$ БПА соотношениями [17],

$$
\left\{\begin{array}{c}
\xi_{X^{\prime} X}=\cos \psi \cos \mu \\
\xi_{X^{\prime} Y}=\sin \psi \cos \mu \\
\xi_{X Z}=\sin \mu \\
\xi_{Y^{\prime} X}=\sin \vartheta \cos \psi \sin \mu-\cos \vartheta \sin \psi ; \\
\xi_{Y^{\prime} Y}=\cos \vartheta \cos \psi+\sin \vartheta \sin \psi \sin \mu ; \\
\xi_{Y^{\prime} Z}=-\sin \vartheta \cos \mu \\
\xi_{Z^{\prime} X}=-\sin \vartheta \sin \psi-\cos \vartheta \cos \psi \sin \mu \\
\xi_{Z^{\prime} Y}=\sin \vartheta \cos \psi-\cos \vartheta \sin \psi \sin \mu \\
\xi_{Z^{\prime} Z}=\cos \vartheta \cos \mu
\end{array}\right.
$$

По вычисленным значениям квадратных матриц $\mathbf{S}_{\text {нзп }}$ и $\mathbf{S}_{\mathrm{cв}}$ из формулы (12) получаем сле-

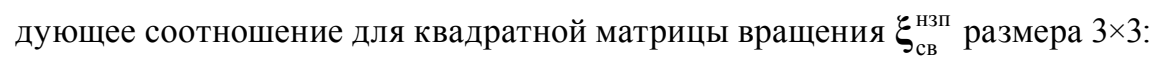

$$
\xi_{\mathrm{cв}}^{\text {нзп }}=\left(\begin{array}{lll}
\xi_{X^{\prime} X} & \xi_{Y^{\prime} X} & \xi_{Z^{\prime} X} \\
\xi_{X^{\prime} Y} & \xi_{Y^{\prime} Y} & \xi_{Z^{\prime} Y} \\
\xi_{X^{\prime} Z} & \xi_{Y^{\prime} Z} & \xi_{Z^{\prime} Z}
\end{array}\right)=\mathbf{S}_{\mathrm{H} 3 \Pi} \mathbf{S}_{\mathrm{cB}}^{-1}
$$

где $\mathbf{S}_{\text {св }}^{-1}$ - матрица, обратная матрице $\mathbf{S}_{\mathrm{cв}}$.

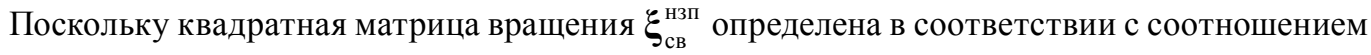
(15), то тем самым однозначно с использованием соотношений (15) определяют углы курса $\psi$, тангажа $\mu$ и крена $\vartheta$ БПА. Например, угол тангажа $\mu$ определяют с использованием значения параметра $\xi_{X^{\prime} Z}$ в виде $\mu=\arcsin \xi_{X^{\prime} Z}$, а углы курса $\psi$ и крена $\vartheta-$ с использованием значений параметров $\xi_{X^{\prime} X}, \xi_{X^{\prime} Y}, \xi_{Y^{\prime} Z}, \xi_{Z^{\prime} Z}($ если $\cos \mu \neq 0)$ или значений параметров $\xi_{Y^{\prime} Z}, \xi_{Z^{\prime} X}, \xi_{Y^{\prime} Y}, \xi_{Z^{\prime} Y}($ в противном случае).

Математические особенности определения пространственного положения БПА. Несмотря на внешнюю простоту системы уравнений (4), она имеет ряд особенностей, связанных с описанием всех ее решений относительно неизвестных значений параметров $\ell_{1}, \ell_{2}$ и $\ell_{3}$ для разных случаев расположения в пространстве БПА и радиоориентиров, требующих обширного математического исследования. С прикладной точки зрения более практичным выглядит подход, заключающийся в разработке приближенного метода решения системы уравнений (4), учитывающего структуру возможных решений, полученную с использованием априорной информации относительно области возможных значений искомых параметров. Без понимания такой структуры возможных решений системы уравнений (4) гораздо сложнее разрабатывать 
эффективные приближенные методы, позволяющие контролировать погрешности входных данных и промежуточных вычислений.

Систему трех уравнений (4) относительно трех неизвестных значений длин боковых ребер $\ell_{1}, \ell_{2}$ и $\ell_{3}$ треугольной пирамиды (см. рис. 1) можно свести в общем случае к уравнению четвертой степени относительно одной переменной. Для пояснения этой возможности необходимо указать на следующие свойства решений исходной системы уравнений (4).

Во-первых, если $\left(\ell_{1}, \ell_{2}, \ell_{3}\right)$ - решение, то, очевидно, $\left(-\ell_{1},-\ell_{2},-\ell_{3}\right)$ тоже решение. Кроме того, хотя решение с отрицательными значениями ребер не «физично», оно оказывается связанным с аналогичной задачей для тех же точек $M_{1}, M_{2}, M_{3}$, но с набором углов $\alpha_{12}, \alpha_{13}$ и $\alpha_{23}$ дающих те же значения по модулю косинусов. Например, если $\left(-\ell_{1}, \ell_{2}, \ell_{3}\right)-$ одно из решений для набора углов $\left(\alpha_{12}, \alpha_{13}, \alpha_{23}\right)$, то $\left(\ell_{1}, \ell_{2}, \ell_{3}\right)$ - решение для углов $\left(\pi-\alpha_{12}, \pi-\alpha_{13}, \alpha_{23}\right)$.

Во-вторых, если считать все возможные решения, в том числе с нулевыми и отрицательными значениями длин боковых ребер $\ell_{1}, \ell_{2}$ и $\ell_{3}$, то их получится не более восьми. С учетом описанной выше симметрии количество положительных решений не превышает четырех.

B-третьих, каждое конкретное решение $\left(\ell_{1}, \ell_{2}, \ell_{3}\right)$ непрерывно зависит от набора углов $\left(\alpha_{12}, \alpha_{13}, \alpha_{23}\right)$.

В-четвертых, систему трех уравнений (4) относительно трех неизвестных значений длин боковых ребер $\ell_{1}, \ell_{2}$ и $\ell_{3}$ треугольной пирамиды $M_{0} M_{1} M_{2} M_{3}$ (см. рис. 1) можно интерпретировать следующим образом. В каждой из боковых треугольных граней $M_{0} M_{i} M_{j}$ треугольной пирамиды $M_{0} M_{1} M_{2} M_{3}$ известна длина $d_{i j}$ стороны $M_{i} M_{j}$ треугольника и угол $\alpha_{i j}$, находящийся напротив указанной стороны $M_{i} M_{j}$, где $1 \leq i<j \leq 3$. Следовательно, для каждой из боковых треугольных граней $M_{0} M_{i} M_{j}$ известен и соответствующий радиус $R_{d_{i j}}$ окружности, описанной около боковой грани $M_{0} M_{i} M_{j}$, причем отрезок $M_{i} M_{j}$, являющийся хордой окружности, делит окружность на две дуги, имеющие в общем случае разные длины. Искомая точка $M_{0}$ треугольной пирамиды $M_{0} M_{1} M_{2} M_{3}$ (см. рис. 1) в зависимости от величины угла $\alpha_{i j}$, который может быть острым или тупым, может располагаться соответственно на большей или меньшей дуге окружности, описанной около боковой грани $M_{0} M_{i} M_{j}$. Для каждой из трех боковых треугольных граней $M_{0} M_{i} M_{j}$ вышепомянутая окружность не единственна и образует семейство окружностей, которое описывается как множество точек, находящихся на поверхности, образованной вращением окружности с радиусом $R_{d_{i j}}$ вокруг оси $M_{i} M_{j}$, лежащей в плоскости этой окружности и в отличие от обычного («открытого») тора пересекающей ее. При этом центр окружности, описанной около боковой грани $M_{0} M_{i} M_{j}$, вращаемой вокруг оси $M_{i} M_{j}$, описывает окружность с центром в середине стороны $M_{i} M_{j}$ основания $M_{1} M_{2} M_{3}$ треугольной пирамиды $M_{0} M_{1} M_{2} M_{3}$ и радиусом, меньшим, чем $R_{d_{i j}}$ и равным $\sqrt{R_{d_{i j}}^{2}-\frac{d_{i j}^{2}}{4}}$. Поэтому вышеупомянутая поверхность представляет собой «закрытый» тор (тор без отверстия в центре), часть внешних границ которого, образованная вращением меньшей дуги окружности с радиусом $R_{d_{i j}}$ располагается внутри его внешних границ, образованных вращением большей дуги окружности с радиусом $R_{d_{i j}}$. Внешний вид «закрытого» тора, образованного вращением окружности с радиусом $R_{d_{i j}}$ вокруг оси $M_{i} M_{j}$, являющейся хордой окружности, представлен на рис. 2. 


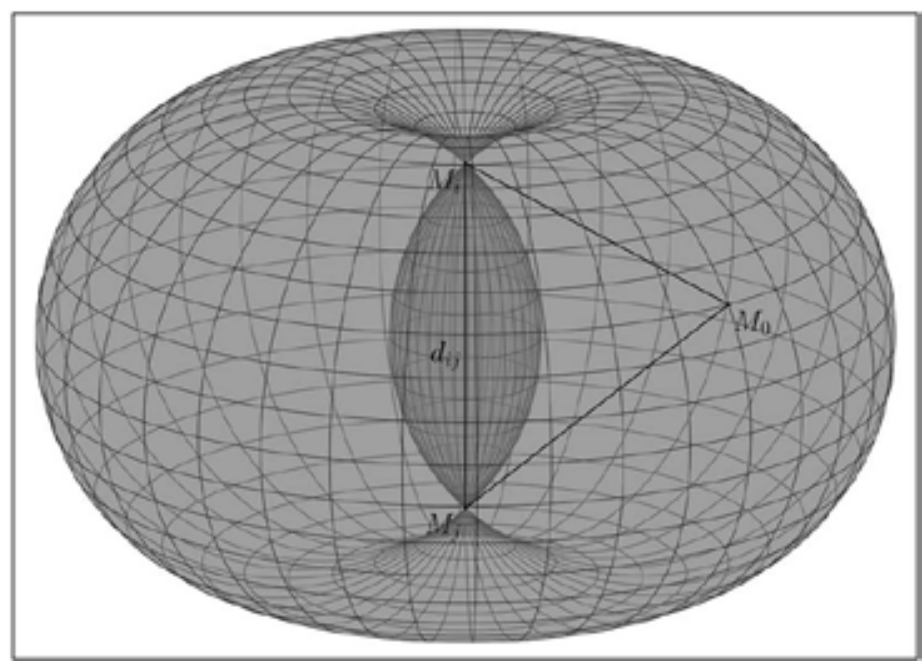

Рис. 2. Внешний вид «закрытого» тора, образованного вращением окружности вокруг ее хорды

Fig. 2. The external view of a "closed" torus formed by the rotation of a circle around its chord

Используя вышеупомянутую геометрическую интерпретацию особенностей описания возможных решений системы уравнений (4) относительно трех неизвестных значений длин боковых ребер $\ell_{1}, \ell_{2}$ и $\ell_{3}$ треугольной пирамиды $M_{0} M_{1} M_{2} M_{3}$ (см. рис. 1), можно составить эквивалентную системам уравнений (4) и (5) систему трех уравнений относительно трех неизвестных координат $x, y$ и $z$ точки $M_{0}(x, y, z)$ расположения ФЦ БПА, обеспечивающую возможность определения координат ФЦ БПА без необходимости определения длин боковых ребер $\ell_{1}, \ell_{2}$ и $\ell_{3}$ треугольной пирамиды. Для этого обозначим точками $M_{i j}\left(x_{i j}, y_{i j}, z_{i j}\right)$ середины ребер $M_{i} M_{j}$ основания $M_{1} M_{2} M_{3}$ треугольной пирамиды $M_{0} M_{1} M_{2} M_{3}$, координаты $x_{i j}, y_{i j}$ и $z_{i j}$ которых определяются в соответствии с соотношениями

$$
x_{i j}=\frac{x_{i}+x_{j}}{2} ; y_{i j}=\frac{y_{i}+y_{j}}{2} ; z_{i j}=\frac{z_{i}+z_{j}}{2},
$$

где $1 \leq i<j \leq 3$.

Тогда систему трех уравнений относительно трех неизвестных координат $x, y$ и $z$ точки $M_{0}(x, y, z)$ расположения ФЦ БПА можно представить в виде

$$
\left\{\begin{array}{l}
\left(\left(x-x_{12}\right)^{2}+\left(y-y_{12}\right)^{2}+\left(z-z_{12}\right)^{2}-\frac{d_{12}^{2}}{4}\right)^{2}-\left(\left(x-x_{12}\right)^{2}+\left(y-y_{12}\right)^{2}\right) d_{12}^{2} \operatorname{ctg}^{2} \alpha_{12}=0 \\
\left(\left(x-x_{13}\right)^{2}+\left(y-y_{13}\right)^{2}+\left(z-z_{13}\right)^{2}-\frac{d_{13}^{2}}{4}\right)^{2}-\left(\left(x-x_{13}\right)^{2}+\left(y-y_{13}\right)^{2}\right) d_{13}^{2} \operatorname{ctg}^{2} \alpha_{13}=0 \\
\left(\left(x-x_{23}\right)^{2}+\left(y-y_{23}\right)^{2}+\left(z-z_{23}\right)^{2}-\frac{d_{23}^{2}}{4}\right)^{2}-\left(\left(x-x_{23}\right)^{2}+\left(y-y_{23}\right)^{2}\right) d_{23}^{2} \operatorname{ctg}^{2} \alpha_{23}=0 .
\end{array}\right.
$$

Для определения областей пространства, в которых совокупность расстояний $\ell_{1}, \ell_{2}$ и $\ell_{3}$ от ФЦ БПА до трех радиоориентиров является однозначной, рассмотрим схему размещения в 
пространстве трех радиоориентиров и фазового центра БПА, приведенную на рис.1. Исходя из геометрического представления задачи нахождения трех неизвестных значений длин боковых ребер $\ell_{1}, \ell_{2}$ и $\ell_{3}$ треугольной пирамиды $M_{0} M_{1} M_{2} M_{3}$, можно утверждать, что если каждый угол $\alpha_{i j}$ боковой грани $M_{0} M_{i} M_{j}$ больше соответствующего ему угла основания $\angle M_{i} M_{k} M_{j}, 1 \leq k \neq i$, $j \leq 3$, то решение единственно, а проекция точки $M_{0}(x, y, z)$ расположения ФЦ БПА на плоскость треугольника $M_{1} M_{2} M_{3}$ находится внутри треугольника основания $M_{1} M_{2} M_{3}$. В случае, когда угол $\alpha_{i j}$ боковой грани $M_{0} M_{i} M_{j}$ оказывается равным соответствующему ему углу основания $\angle M_{i} M_{k} M_{j}$, возникает решение с нулевым ребром. При уменьшении угла $\alpha_{i j}$ боковой грани $M_{0} M_{i} M_{j}$ (например, при вертикальном подъеме БПА относительно плоскости треугольника $M_{1} M_{2} M_{3}$ ), в силу непрерывности, все значения боковых ребер $\ell_{1}, \ell_{2}$ и $\ell_{3}$ треугольной пирамиды $M_{0} M_{1} M_{2} M_{3}$ являются ненулевыми и положительными.

Особенности нахождения аналитического решения системы уравнений (4) относительно искомых значений параметров $\ell_{1}, \ell_{2}$ и $\ell_{3}$ продемонстрируем на основе частного случая, когда основание $M_{1} M_{2} M_{3}$ треугольной пирамиды $M_{0} M_{1} M_{2} M_{3}$ (см. рис. 1) представляет собой равносторонний треугольник со стороной $d=d_{12}=d_{13}=d_{23}$. Заметим, что используемые при этом приемы в целом применимы и для общего случая. При условии $d=d_{12}=d_{13}=d_{23}$ и замены переменных $\ell_{2}=b \ell_{1}, \ell_{3}=c \ell_{1}$ (случай $\ell_{1}=0$ следует рассмотреть отдельно) система уравнений (4) примет вид

$$
\left\{\begin{array}{c}
\ell_{1}^{2}\left(1-2 b \cos \alpha_{12}+b^{2}\right)=d^{2} ; \\
\ell_{1}^{2}\left(1-2 c \cos \alpha_{13}+c^{2}\right)=d^{2} \\
\ell_{1}^{2}\left(c^{2}-2 b c \cos \alpha_{23}+b^{2}\right)=d^{2} .
\end{array}\right.
$$

Если разделить первое уравнение системы (18) на второе, далее из суммы первого и второго уравнения вычесть третье уравнение системы (18) и, наконец, результат поделить на первое уравнение системы (18), то относительно двух неизвестных параметров $b$ и $c$ получим следующую систему из двух уравнений:

$$
\left\{\begin{array}{l}
b^{2}-2 b \cos \alpha_{12}=c^{2}-2 c \cos \alpha_{13} \\
2 c\left(b \cos \alpha_{23}-\cos \alpha_{13}\right)=b^{2}-1
\end{array}\right.
$$

Из второго уравнения системы уравнений (19) выразим параметр $c$ через неизвестный параметр $b$ (случай $\mathrm{b}= \pm 1$ требует отдельного рассмотрения), подставим полученное соотношение в первое уравнение системы уравнений (19) и относительно неизвестного параметра $b$ получим уравнение

$$
\begin{aligned}
& \left(1-4 \cos ^{2} \alpha_{23}\right) b^{4}+4 \cos \alpha_{23}\left(\cos \alpha_{23}+2 \cos \alpha_{12} \cos \alpha_{23}\right) b^{3}-2\left(1+8 \cos \alpha_{12} \cos \alpha_{13} \cos \alpha_{23}\right) b^{2}+\ldots \rightarrow \\
& \rightarrow \ldots+4 \cos \alpha_{13}\left(\cos \alpha_{23}+2 \cos \alpha_{12} \cos \alpha_{13}\right) b+1-4 \cos ^{2} \alpha_{13}=0 .
\end{aligned}
$$

Для уравнения (20) значения угла $\alpha_{23}$, при которых $\cos \alpha_{23}=\frac{1}{2}$, являются особыми. При таких значениях угла $\alpha_{23}$ порядок уравнения (20) понижается с четырех до трех. Кроме того, значения корня $b= \pm 1$ приводят к другим частным случаям. Эти обстоятельства не позволяют в общем случае использовать уравнение (20) для численного решения исходной системы уравне- 
ний (18), поскольку при приближении к вышеупомянутым вырожденным случаям нарушается устойчивость искомых решений.

Следует отметить, что можно существенно упростить решение системы уравнений (5) путем введения локальной системы координат (ЛСК) $\Sigma_{\text {л }}=\left\{O^{\prime \prime}, X^{\prime \prime}, Y^{\prime \prime}, Z^{\prime \prime}\right\}$, представляющей собой левую пространственную прямоугольную декартовую систему координат, начало $O^{\prime \prime}$ которой находится в точке пересечения медиан основания $M_{1} M_{2} M_{3}$ треугольной пирамиды $M_{0} M_{1} M_{2} M_{3}$, координаты $x_{O^{\prime \prime}}, y_{O^{\prime \prime}}$ и $z_{O^{\prime \prime}}$ которой в НЗСК $\Sigma_{\text {нз }}=\{O, X, Y, Z\}$ определяются соотношениями ось абсцисс $O^{\prime \prime} X^{\prime \prime}$ которой, находящаяся в плоскости основания $M_{1} M_{2} M_{3}$ треугольной пирамиды $M_{0} M_{1} M_{2} M_{3}$, проходит через точку $M_{1}$ размещения первого радиоориентира, ось аппликат $O^{\prime \prime} Z^{\prime \prime}$ которой перпендикулярна плоскости основания $M_{1} M_{2} M_{3}$ с положительным направлением, образующим острый угол с осью аппликат $O Z$ НЗСК $\Sigma_{\text {нз }}=\{O, X, Y, Z\}$, а ось ординат $O^{\prime \prime} Y^{\prime \prime}$ которой, находящаяся в плоскости основания $M_{1} M_{2} M_{3}$ треугольной пирамиды $M_{0} M_{1} M_{2} M_{3}$, дополняет систему до левой пространственной прямоугольной декартовой системы координат.

$$
x_{O^{\prime \prime}}=\frac{x_{1}+x_{2}+x_{3}}{3} ; y_{O^{\prime \prime}}=\frac{y_{1}+y_{2}+y_{3}}{3} ; z_{O^{\prime \prime}}=\frac{z_{1}+z_{2}+z_{3}}{3} \text {. }
$$

Пусть $\mathbf{r}_{O^{\prime \prime}}=\left(x_{O^{\prime \prime}}, y_{O^{\prime \prime}}, z_{O^{\prime \prime}}\right)$ - радиус-вектор точки $O^{\prime \prime}$ в НЗСК $\Sigma_{\text {нз }}=\{O, X, Y, Z\}$, а вектор нормали $\mathbf{n}$ к плоскости треугольника $M_{1} M_{2} M_{3}$ определяется следующим образом:

$$
\mathbf{n}=\left(\mathbf{r}_{2}-\mathbf{r}_{1}\right) \times\left(\mathbf{r}_{3}-\mathbf{r}_{1}\right)=\left|\begin{array}{ccc}
\mathbf{s}_{\mathrm{H} 31} & \mathbf{s}_{\mathrm{H} 32} & \mathbf{s}_{\mathrm{н} 33} \\
x_{2}-x_{1} & y_{2}-y_{1} & z_{2}-z_{1} \\
x_{3}-x_{1} & y_{3}-y_{1} & z_{3}-z_{1}
\end{array}\right|,
$$

где $\mathbf{s}_{\mathrm{H} 31}=(1,0,0), \mathbf{s}_{\mathrm{H} 32}=(0,1,0), \mathbf{s}_{\mathrm{H} 33}=(0,0,1)-$ единичные орты в НЗСК. Если угол между положи-

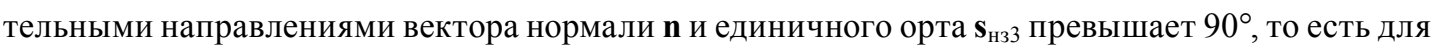
скалярного произведения выполняется соотношение $\mathbf{n} \cdot \mathbf{s}_{\text {нз3 }}<0$, то вектор $\mathbf{n}$ заменяется на $-\mathbf{n}$.

Определим единичные орты $\mathbf{s}_{\text {л1 }}, \mathbf{s}_{\text {л2 }}$ и $\mathbf{s}_{\text {л3 }}$ ЛСК $\Sigma_{\text {л }}=\left\{O^{\prime \prime}, X^{\prime \prime}, Y^{\prime \prime}, Z^{\prime \prime}\right\}$ в виде следующих соотношений:

$$
\mathbf{s}_{\text {Л1 }}=\frac{\mathbf{r}_{1}-\mathbf{r}_{O^{\prime \prime}}}{\left|\mathbf{r}_{1}-\mathbf{r}_{O^{\prime \prime}}\right|} ; \mathbf{s}_{\text {Л } 2}=\frac{\mathbf{s}_{\text {л1 }} \times \mathbf{s}_{\text {Л3 }}}{\left|\mathbf{s}_{\text {л1 }} \times \mathbf{s}_{\text {Л3 }}\right|} ; \mathbf{s}_{\text {Л3 }}=\frac{\mathbf{n}}{|\mathbf{n}|} .
$$

Отметим, что порядок сомножителей в векторном произведении $\mathbf{s}_{\text {л1 }} \times \mathbf{s}_{\text {л3 }}$, определяющем в соответствии с (23) единичный орт $\mathbf{s}_{л 2}$, выбран таким образом, чтобы упорядоченная совокупность единичных ортов $\mathbf{s}_{\text {л1 }}, \mathbf{s}_{\text {л2 }}$ и $\mathbf{s}_{\text {л3 }}$ ЛСК $\Sigma_{\text {л }}=\left\{O^{\prime \prime}, X^{\prime \prime}, Y^{\prime \prime}, Z^{\prime \prime}\right\}$ образовывала левую тройку векторов.

Пусть $\mathbf{r}$ - радиус вектор точки $M_{0}$ расположения ФЦ БПА, имеющей координаты $(x, y, z)$ в НЗСК и координаты $\left(x^{\prime \prime}, y^{\prime \prime}, z^{\prime \prime}\right)$ в ЛСК. Тогда с учетом (21)-(23) справедливо векторное соотношение

$$
\mathbf{r}=x \mathbf{s}_{\mathrm{н} 31}+y \mathbf{s}_{\mathrm{н} 32}+z \mathbf{s}_{\mathrm{H} 33}=\mathbf{r}_{O^{\prime \prime}}+x^{\prime \prime} \mathbf{s}_{\text {л1 }}+y^{\prime \prime} \mathbf{s}_{\text {л2 }}+z^{\prime \prime} \mathbf{s}_{\text {л3 }} .
$$

Из векторного соотношения (24) с учетом (21)-(23) можно получить следующие соотношения для определения координат $(x, y, z)$ точки $M_{0}$ в НЗСК по полученным (заданным) значениям координат $\left(x^{\prime \prime}, y^{\prime \prime}, z^{\prime \prime}\right)$ этой же точки в ЛСК: 


$$
\left\{\begin{array}{l}
x=x_{O^{\prime \prime}}+x^{\prime \prime}\left(\mathbf{s}_{\text {л1 }} \cdot \mathbf{s}_{\mathrm{H} 31}\right)+y^{\prime \prime}\left(\mathbf{s}_{\text {л2} 2} \cdot \mathbf{s}_{\mathrm{H} 31}\right)+z^{\prime \prime}\left(\mathbf{s}_{\text {л3 }} \cdot \mathbf{s}_{\mathrm{H} 31}\right) ; \\
y=y_{O^{\prime \prime}}+x^{\prime \prime}\left(\mathbf{s}_{\text {л1 }} \cdot \mathbf{s}_{\mathrm{H} 32}\right)+y^{\prime \prime}\left(\mathbf{s}_{\text {л2} 2} \cdot \mathbf{s}_{\mathrm{H} 32}\right)+z^{\prime \prime}\left(\mathbf{s}_{\text {л3 }} \cdot \mathbf{s}_{\mathrm{H} 32}\right) \\
z=z_{O^{\prime \prime}}+x^{\prime \prime}\left(\mathbf{s}_{\text {л1 }} \cdot \mathbf{s}_{\mathrm{H} 33}\right)+y^{\prime \prime}\left(\mathbf{s}_{\text {л2 }} \cdot \mathbf{s}_{\mathrm{H} 33}\right)+z^{\prime \prime}\left(\mathbf{s}_{\text {л3 }} \cdot \mathbf{s}_{\mathrm{H} 33}\right),
\end{array}\right.
$$

и следующие соотношения для определения координат $\left(x^{\prime \prime}, y^{\prime \prime}, z^{\prime \prime}\right)$ точки $M_{0}$ в ЛСК по полученным (заданным) значениям координат $(x, y, z)$ этой же точки в НЗСК:

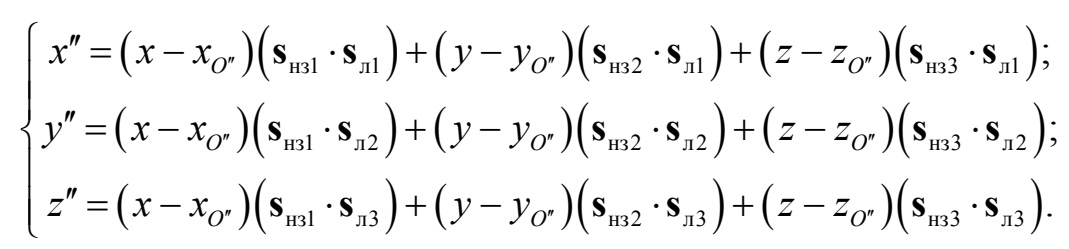

Пространственное положение точек $M_{i}$ размещения $i$-х радиоориентиров в вышеупомянутой ЛСК $\Sigma_{\text {л }}=\left\{O^{\prime \prime}, X^{\prime \prime}, Y^{\prime \prime}, Z^{\prime \prime}\right\}$ будем характеризовать радиус-векторами $\mathbf{r}_{i}=\left(x_{i}^{\prime \prime}, y_{i}^{\prime \prime}, 0\right)$, находящимися в плоскости основания $M_{1} M_{2} M_{3}$ треугольной пирамиды $M_{0} M_{1} M_{2} M_{3}$, где $i=1,2,3$. Тогда с учетом (25) и (26) и определенности выбора положительного направления оси абсцисс $O^{\prime \prime} X^{\prime \prime}$ ЛСК $\Sigma_{\text {л }}=\left\{O^{\prime \prime}, X^{\prime \prime}, Y^{\prime \prime}, Z^{\prime \prime}\right\}$, проходящего через точку $M_{1}$ размещения первого радиоориентира, при котором $y_{1}^{\prime \prime}=0$, система уравнений (5) для неизвестных координат $x^{\prime \prime}, y^{\prime \prime}$ и $z^{\prime \prime}$ точки $M_{0}\left(x^{\prime \prime}, y^{\prime \prime}, z^{\prime \prime}\right)$ расположения ФЦ БПА в ЛСК $\Sigma_{\text {л }}=\left\{O^{\prime \prime}, X^{\prime \prime}, Y^{\prime \prime}, Z^{\prime \prime}\right\}$ может быть представлена в виде

$$
\left\{\begin{array}{l}
\left(x^{\prime \prime}-x_{1}^{\prime \prime}\right)^{2}+\left(y^{\prime \prime}\right)^{2}+\left(z^{\prime \prime}\right)^{2}=\ell_{1}^{2} \\
\left(x^{\prime \prime}-x_{2}^{\prime \prime}\right)^{2}+\left(y^{\prime \prime}-y_{2}^{\prime \prime}\right)^{2}+\left(z^{\prime \prime}\right)^{2}=\ell_{2}^{2} \\
\left(x^{\prime \prime}-x_{3}^{\prime \prime}\right)^{2}+\left(y^{\prime \prime}-y_{3}^{\prime \prime}\right)^{2}+\left(z^{\prime \prime}\right)^{2}=\ell_{3}^{2}
\end{array}\right.
$$

При этом система из двух уравнений относительно неизвестных значений координат $x^{\prime \prime}$ и $y^{\prime \prime}$ точки $M_{0}$ расположения ФЦ БПА в ЛСК $\Sigma_{\text {Л }}=\left\{O^{\prime \prime}, X^{\prime \prime}, Y^{\prime \prime}, Z^{\prime \prime}\right\}$, полученная аналогичным образом, как и система уравнений (6), с учетом (27) может быть представлена в виде

$$
\left\{\begin{array}{l}
2 x^{\prime \prime}\left(x_{1}^{\prime \prime}-x_{2}^{\prime \prime}\right)-2 y^{\prime \prime} y_{2}^{\prime \prime}=\ell_{2}^{2}-\ell_{1}^{2}+\left(x_{1}^{\prime \prime}\right)^{2}-\left(x_{2}^{\prime \prime}\right)^{2}-\left(y_{2}^{\prime \prime}\right)^{2} \\
2 x^{\prime \prime}\left(x_{1}^{\prime \prime}-x_{3}^{\prime \prime}\right)-2 y^{\prime \prime} y_{3}^{\prime \prime}=\ell_{3}^{2}-\ell_{1}^{2}+\left(x_{1}^{\prime \prime}\right)^{2}-\left(x_{3}^{\prime \prime}\right)^{2}-\left(y_{3}^{\prime \prime}\right)^{2}
\end{array}\right.
$$

Согласно правилу Крамера решение системы уравнений (28) относительно координат $x^{\prime \prime}$ и $y^{\prime \prime}$ точки $M_{0}$ расположения ФЦ БПА в ЛСК, в отличие от (7) не зависящее от неизвестного значения координаты $z^{\prime \prime}$, определяется соотношениями

где

$$
x^{\prime \prime}=\frac{\left|\begin{array}{ll}
A_{\text {л }} & -2 y_{2}^{\prime \prime} \\
B_{\text {л }} & -2 y_{3}^{\prime \prime}
\end{array}\right|}{2\left(y_{2}^{\prime \prime}\left(x_{1}^{\prime \prime}-x_{3}^{\prime \prime}\right)-y_{3}^{\prime \prime}\left(x_{1}^{\prime \prime}-x_{2}^{\prime \prime}\right)\right)} ; y^{\prime \prime}=\frac{\left|\begin{array}{ll}
2\left(x_{1}^{\prime \prime}-x_{2}^{\prime \prime}\right) & A_{\text {л }} \\
2\left(x_{1}^{\prime \prime}-x_{3}^{\prime \prime}\right) & B_{\text {л }}
\end{array}\right|}{2\left(y_{2}^{\prime \prime}\left(x_{1}^{\prime \prime}-x_{3}^{\prime \prime}\right)-y_{3}^{\prime \prime}\left(x_{1}^{\prime \prime}-x_{2}^{\prime \prime}\right)\right)},
$$

$$
A_{\text {л }}=\ell_{2}^{2}-\ell_{1}^{2}+\left(x_{1}^{\prime \prime}\right)^{2}-\left(x_{2}^{\prime \prime}\right)^{2}-\left(y_{2}^{\prime \prime}\right)^{2}, B_{\text {л }}=\ell_{3}^{2}-\ell_{1}^{2}+\left(x_{1}^{\prime \prime}\right)^{2}-\left(x_{3}^{\prime \prime}\right)^{2}-\left(y_{3}^{\prime \prime}\right)^{2} .
$$


Из первого уравнения системы (27) с учетом полученных в соответствии с (29) значений $x^{\prime \prime}$ и $y^{\prime \prime}$ неизвестное значение координаты $z^{\prime \prime}$ точки $M_{0}\left(x^{\prime \prime}, y^{\prime \prime}, z^{\prime \prime}\right)$ расположения ФЦ БПА в ЛСК $\Sigma_{\text {л }}=\left\{O^{\prime \prime}, X^{\prime \prime}, Y^{\prime \prime}, Z^{\prime \prime}\right\}$ определяется в соответствии с соотношением

$$
\left(z^{\prime \prime}\right)^{2}=\ell_{1}^{2}-\left(x^{\prime \prime}-x_{1}^{\prime \prime}\right)^{2}-\left(y^{\prime \prime}\right)^{2} .
$$

Определение координат $(x, y, z)$ точки $M_{0}$ в НЗСК $\Sigma_{\text {нз }}=\{O, X, Y, Z\}$ по полученным в соответствии с (29) и (30) значениям координат $\left(x^{\prime \prime}, y^{\prime \prime}, z^{\prime \prime}\right)$ этой же точки в ЛСК $\Sigma_{\text {л }}=\left\{O^{\prime \prime}, X^{\prime \prime}, Y^{\prime \prime}, Z^{\prime \prime}\right\}$ осуществляется в соответствии с соотношением (25).

Способы определения дальности до радиоориентиров по результатам их азимутально-угломестного радиопеленгования. Решение системы уравнений (4) относительно неизвестных значений длин $\ell_{1}, \ell_{2}$ и $\ell_{3}$ боковых ребер треугольной пирамиды $M_{0} M_{1} M_{2} M_{3}$ (см. рис. 1), соответствующих дальностям от БПА до радиоориентиров, с помощью прямых аналитических методов, как было сказано ранее, сводится к поиску корней многочлена четвертой степени, один из вариантов которого определяется соотношением (20). Коэффициент при старшей степени может быть малым или даже вырождаться, что является причиной неустойчивости поиска корней вышеупомянутого многочлена. Поэтому более удобным с практической точки зрения представляется применение приближенного метода решения. Будем использовать метод Ньютона для нелинейных систем [20]. Этот метод наиболее эффективен в случае, когда имеется априорно известное начальное приближение для искомого решения. Обратная матрица, возникающая при реализации метода Ньютона, выписывается явно. Нет никаких других операций над данными, кроме арифметических, поскольку значения $\cos \alpha_{i j}$ вычисляют в соответствии с соотношением (2) один раз по результатам измерений азимутов $\alpha_{i}$ и углов места $\varepsilon_{i}$ при одновременном азимутально-угломестном радиопеленговании $i$-х радиоориентиров с использованием БПА. Для получения требуемой точности вычислений искомых значений длин $\ell_{1}, \ell_{2}$ и $\ell_{3}$ достаточно сделать 5-6 итераций. Это обеспечивает проведение расчетов в режиме реального времени. Решение системы уравнений (4), как было ранее отмечено, не является однозначным. Вместе с тем в условиях наличия априорно известной информации о ранее упомянутых закономерностях и условиях возникновения паразитных решений можно заранее рассчитать рекомендуемые области размещения БПА относительно радиоориентиров, чтобы метод Ньютона при решении системы уравнений (4) относительно неизвестных значений параметров $\ell_{1}, \ell_{2}$ и $\ell_{3}$ сходился к истинным значениям искомых параметров.

Для решения системы уравнений (4) методом Ньютона представим ее в векторной форме с использованием вектора $\mathbf{F}(\mathbf{L})$ в виде соотношения

$$
\mathbf{F}(\mathbf{L})=\left(\begin{array}{l}
F_{1}(\mathbf{L}) \\
F_{2}(\mathbf{L}) \\
F_{3}(\mathbf{L})
\end{array}\right)=\left(\begin{array}{l}
\ell_{1}^{2}+\ell_{2}^{2}-\ell_{1} \ell_{2} \cos \alpha_{12}-d_{12}^{2} \\
\ell_{1}^{2}+\ell_{3}^{2}-\ell_{1} \ell_{3} \cos \alpha_{13}-d_{13}^{2} \\
\ell_{2}^{2}+\ell_{3}^{2}-\ell_{2} \ell_{3} \cos \alpha_{23}-d_{23}^{2}
\end{array}\right)=\left(\begin{array}{l}
0 \\
0 \\
0
\end{array}\right),
$$

где $\mathbf{L}=\left(\ell_{1}, \ell_{2}, \ell_{3}\right)^{\mathrm{T}}$ - вектор-столбец неизвестных параметров $\ell_{1}, \ell_{2}$ и $\ell_{3} ; F_{1}=F_{1}(\mathbf{L}), F_{2}=F_{2}(\mathbf{L})$ и $F_{3}=F_{3}(\mathbf{L})$ - компоненты вектор-столбца $\mathbf{F}(\mathbf{L})$, задаваемые в соответствии с системой уравнений (4). 
Обозначим через $\mathbf{D F}(\mathbf{L})$ квадратную матрицу размера $3 \times 3$, элементами которой являются частные производные $\frac{\partial F_{i}}{\partial \ell_{j}}$ (где $i=1,2,3 ; j=1,2,3$ ) компонент $F_{i}$ вектор-столбца $\mathbf{F}(\mathbf{L})$, определяемую с учетом (31) соотношением

$$
\mathbf{D F}(\mathbf{L})=2\left(\begin{array}{ccc}
\ell_{1}-\ell_{2} \cos \alpha_{12} & \ell_{2}-\ell_{1} \cos \alpha_{12} & 0 \\
\ell_{1}-\ell_{3} \cos \alpha_{13} & 0 & \ell_{3}-\ell_{2} \cos \alpha_{13} \\
0 & \ell_{2}-\ell_{3} \cos \alpha_{23} & \ell_{3}-\ell_{2} \cos \alpha_{23}
\end{array}\right)
$$

Кроме того, обозначим через $\mathbf{L}^{(m)}=\left(\ell_{1}^{(m)}, \ell_{2}^{(m)}, \ell_{3}^{(m)}\right)^{\mathrm{T}}$ вектор-столбец параметров $\ell_{1}^{(m)}, \ell_{2}^{(m)}$ и $\ell_{3}^{(m)}$, соответствующих $m$-й итерации решения системы уравнений (4) методом Ньютона, где $m=0,1,2, \ldots$ - порядковый номер итерации. Тогда вектор-столбец $\mathbf{L}^{(m+1)}=\left(\ell_{1}^{(m+1)}, \ell_{2}^{(m+1)}, \ell_{3}^{(m+1)}\right)^{\mathrm{T}}$ дальностей от БПА до радиоориентиров $\ell_{1}^{(m+1)}, \ell_{2}^{(m+1)}$ и $\ell_{3}^{(m+1)}$, определяемых на $(m+1)$-й итерации решения системы уравнений (4) методом Ньютона [20], с учетом (31) и (32) определяется соотношением

$$
\mathbf{L}^{(m+1)}=\mathbf{L}^{(m)}-\left(\mathbf{D F}\left(\mathbf{L}^{(m)}\right)\right)^{-1} \mathbf{F}\left(\mathbf{L}^{(m)}\right)
$$

где $\left(\mathbf{D F}\left(\mathbf{L}^{(m)}\right)\right)^{-1}$ - матрица, обратная матрице $\mathbf{D F}\left(\mathbf{L}^{(m)}\right)$, соответствующей $m$-й итерации решения системы уравнений (4), определяемая с учетом (32) соотношением

$$
\left(\mathbf{D F}\left(\mathbf{L}^{(m)}\right)\right)^{-1}=\frac{1}{K_{1}^{(m)} K_{4}^{(m)} K_{5}^{(m)}-K_{2}^{(m)} K_{3}^{(m)} K_{6}^{(m)}}\left(\begin{array}{ccc}
K_{4}^{(m)} K_{5}^{(m)} & K_{2}^{(m)} K_{6}^{(m)} & -K_{2}^{(m)} K_{4}^{(m)} \\
K_{3}^{(m)} K_{6}^{(m)} & -K_{1}^{(m)} K_{6}^{(m)} & K_{1}^{(m)} K_{4}^{(m)} \\
-K_{3}^{(m)} K_{5}^{(m)} & K_{1}^{(m)} K_{5}^{(m)} & K_{2}^{(m)} K_{3}^{(m)}
\end{array}\right),
$$

где

$$
\begin{aligned}
& K_{1}^{(m)}=2 \ell_{1}^{(m)}-2 \ell_{2}^{(m)} \cos \alpha_{12} ; K_{2}^{(m)}=2 \ell_{2}^{(m)}-2 \ell_{1}^{(m)} \cos \alpha_{12} ; \\
& K_{3}^{(m)}=2 \ell_{1}^{(m)}-2 \ell_{3}^{(m)} \cos \alpha_{13} ; K_{4}^{(m)}=2 \ell_{3}^{(m)}-2 \ell_{1}^{(m)} \cos \alpha_{13} ; \\
& K_{5}^{(m)}=2 \ell_{2}^{(m)}-2 \ell_{3}^{(m)} \cos \alpha_{23} ; K_{6}^{(m)}=2 \ell_{3}^{(m)}-2 \ell_{2}^{(m)} \cos \alpha_{23} .
\end{aligned}
$$

При условии, что проекция ФЦ БПА на плоскость основания $M_{1} M_{2} M_{3}$ треугольной пирамиды $M_{0} M_{1} M_{2} M_{3}$ находится внутри треугольника $M_{1} M_{2} M_{3}$ и априорной неопределенности положения ФЦ БПА относительно радиоориентиров, в качестве «нулевой» итерации $(m=0)$ однозначного решения системы уравнений (4) методом Ньютона можно выбрать, например, вектор-столбец $\mathbf{L}^{(0)}=\left(\ell_{1}^{(0)}, \ell_{2}^{(0)}, \ell_{3}^{(0)}\right)^{\mathrm{T}}$ дальностей от точки $O^{\prime \prime}$ пересечения медиан основания $M_{1} M_{2} M_{3}$ треугольной пирамиды $M_{0} M_{1} M_{2} M_{3}$ до точек $M_{1}\left(x_{1}, y_{1}, z_{1}\right), M_{2}\left(x_{2}, y_{2}, z_{2}\right)$ и $M_{3}\left(x_{3}, y_{3}, z_{3}\right)$ размещения радиоориентиров, компоненты $\ell_{i}^{(0)}$ которого с учетом (21) определяются соотношением

$$
\ell_{i}^{(0)}=\sqrt{\left(x_{i}-x_{O^{\prime \prime}}\right)^{2}+\left(y_{i}-y_{O^{\prime \prime}}\right)^{2}+\left(z_{i}-z_{O^{\prime \prime}}\right)^{2}},
$$

где $i=1,2,3$.

В последующем при периодически осуществляемом азимутально-угломестном радиопеленговании $i$-х радиоориентиров с использованием БПА и определением соответствующих $i$-м радиоориентирам азимутов $\alpha_{i}$ и углов места $\varepsilon_{i}$ в качестве «нулевой» итерации $(m=0)$ одно- 
значного решения системы уравнений (4) методом Ньютона в соответствии с соотношением (33) выбирается вектор-столбец $\mathbf{L}=\left(\ell_{1}, \ell_{2}, \ell_{3}\right)^{\mathrm{T}}$ дальностей от ФЦ БПА до точек $M_{1}\left(x_{1}, y_{1}, z_{1}\right)$, $M_{2}\left(x_{2}, y_{2}, z_{2}\right)$ и $M_{3}\left(x_{3}, y_{3}, z_{3}\right)$ размещения радиоориентиров, полученных по результатам предыдущего измерения азимутов $\alpha_{i}$ и углов места $\varepsilon_{i}$ радиоориентиров.

Необходимо отметить, что при достижении определенной (критической) высоты $h$ размещения ФЦ БПА относительно плоскости основания $M_{1} M_{2} M_{3}$ треугольной пирамиды $M_{0} M_{1} M_{2} M_{3}$ (см. рис. 1), зависящей от радиуса $R$ окружности, описанной вокруг треугольника $\Delta M_{1} M_{2} M_{3}$, если проекция ФЦ БПА на плоскость основания $M_{1} M_{2} M_{3}$ треугольной пирамиды $M_{0} M_{1} M_{2} M_{3}$ находится внутри треугольника $\Delta M_{1} M_{2} M_{3}$, появляется второе (ложное) решение системы уравнений (4), соответствующее расположению ФЦ БПА в одной из вершин треугольника $\Delta M_{1} M_{2} M_{3}$ (одно из значений длин боковых ребер $\ell_{1}, \ell_{2}$ и $\ell_{3}$ треугольной пирамиды $M_{0} M_{1} M_{2} M_{3}$ становится равным нулю). При дальнейшем увеличении высоты размещения ФЦ БПА относительно плоскости основания $M_{1} M_{2} M_{3}$ треугольной пирамиды $M_{0} M_{1} M_{2} M_{3}$ вышеупомянутое ложное решение системы уравнений (4) определяет положение ФЦ БПА, при котором высота размещения ФЦ БПА относительно плоскости основания $M_{1} M_{2} M_{3}$ увеличивается, а проекция ФЦ БПА на плоскость основания $M_{1} M_{2} M_{3}$ треугольной пирамиды $M_{0} M_{1} M_{2} M_{3}$ находится вне треугольника $\Delta M_{1} M_{2} M_{3}$ и удаляется от вышеупомянутой вершины треугольника $\Delta M_{1} M_{2} M_{3}$. При достижении следующих критических высот появляются второе и третье аналогичные ложные решения системы уравнений (4), соответствующие положениям ФЦ БПА вблизи двух других вершин треугольника $\Delta M_{1} M_{2} M_{3}$. При определенных симметриях вышеупомянутые ложные решения системы уравнений (4) могут появляться парой или даже тройкой. Это зависит от конфигурации треугольника $\Delta M_{1} M_{2} M_{3}$, лежащего в основании треугольной пирамиды $M_{0} M_{1} M_{2} M_{3}$, и траектории движения БПА.

Необходимо отметить, что возможность однозначного решения системы уравнений (4) существует также и в случае, когда проекция ФЦ БПА на плоскость основания $M_{1} M_{2} M_{3}$ треугольной пирамиды $M_{0} M_{1} M_{2} M_{3}$ находится вне треугольника $\Delta M_{1} M_{2} M_{3}$. Структура ложных решений системы уравнений (4), соответственно, также перестраивается. Задача полного описания решений системы уравнений (4) для всех возможных случаев представляется сложной и вряд ли реализуемой в наглядной и удобной для практического использования форме. Гораздо проще проводить расчеты по методике, предложенной выше, для заданной конфигурации размещения радиоориентиров и возможных траекторий движения БПА.

Одним из других способов устранения неоднозначности решения системы уравнений (4) является использование результатов азимутально-угломестного радиопеленгования дополнительного четвертого радиоориентира. Размещение четвертого радиоориентира в произвольном положении относительно основания $M_{1} M_{2} M_{3}$ треугольной пирамиды $M_{0} M_{1} M_{2} M_{3}$ приводит к переопределенной системе уравнений относительно расстояний от ФЦ БПА до радиоориетниров. Фактически вместо одного треугольника в основании $M_{1} M_{2} M_{3}$ треугольной пирамиды $M_{0} M_{1} M_{2} M_{3}$ при добавлении четвертого радиоориентира можно сформировать четыре треугольника. То есть можно определять неоднозначные решения от двух до четырех систем уравнений, аналогичных системе уравнений (4), и выбирать в качестве однозначного совместные решения. Однако с учетом погрешностей азимутально-угломестного радиопеленгования радиоориентиров этот вариант требует отдельного исследования. Вместе с тем, если дополнительный (чет-

$$
-305-
$$


вертый) радиоориентир будет располагаться на одном из ребер основания $M_{1} M_{2} M_{3}$ треугольной пирамиды $M_{0} M_{1} M_{2} M_{3}$, то исходная задача однозначного решения системы уравнений (4) значительно упрощается - решение становится единственным и определяется в виде аналитических соотношений.

Без ограничения общности будем считать, что точка $M_{4}\left(x_{4}, y_{4}, z_{4}\right)$ с заданными известными координатами $x_{4}, y_{4}$ и $z_{4}$ размещена на ребре $M_{1} M_{2}$ основания $M_{1} M_{2} M_{3}$ треугольной пирамиды $M_{0} M_{1} M_{2} M_{3}$, что поясняется схемой точек $M_{0}, M_{1}, M_{2}, M_{3}$ и $M_{4}$ размещения в пространстве соответственно фазового центра БПА и первого, второго, третьего и четвертого радиоориентиров, приведенной на рис. 3, где кроме ранее приведенных обозначений обозначено: $\alpha_{14}=\angle M_{1} M_{0} M_{4}$ - плоский угол при вершине $M_{0}$ между боковыми ребрами $M_{0} M_{1}$ и $M_{0} M_{4}$ треугольной пирамиды $M_{0} M_{1} M_{3} M_{4} ; \alpha_{24}=\angle M_{2} M_{0} M_{4}$ - плоский угол при вершине $M_{0}$ между боковыми ребрами $M_{0} M_{2}$ и $M_{0} M_{4}$ треугольной пирамиды $M_{0} M_{2} M_{3} M_{4}$; $\varphi=\angle M_{0} M_{4} M_{1}-$ угол с вершиной в точке $M_{4}$ между отрезками линий $M_{4} M_{0}$ и $M_{4} M_{1}$, размещенными на боковой грани $M_{0} M_{1} M_{2}$ треугольной пирамиды $M_{0} M_{1} M_{2} M_{3}$. Введем также обозначения отрезков $d_{14}=M_{1} M_{4}$ и $d_{24}=M_{2} M_{4}$ ребра $M_{1} M_{2}$ основания $M_{1} M_{2} M_{3}$ треугольной пирамиды $M_{0} M_{1} M_{2} M_{3}$.

Так как $\sin (\pi-\varphi)=\sin \varphi$, то из треугольников $M_{0} M_{4} M_{1}$ и $M_{0} M_{4} M_{2}$ по теореме синусов можно получить равенства $\frac{d_{14}}{\sin \alpha_{14}}=\frac{\ell_{1}}{\sin \varphi}$ и $\frac{d_{24}}{\sin \alpha_{24}}=\frac{\ell_{2}}{\sin \varphi}$, из которых получаем следующее соотношение:

$$
\frac{\ell_{1} \sin \alpha_{14}}{d_{14}}=\frac{\ell_{2} \sin \alpha_{24}}{d_{24}}=\sin \varphi .
$$

Введем обозначение

$$
\rho=\frac{d_{24} \sin \alpha_{14}}{d_{14} \sin \alpha_{24}} .
$$

Тогда с учетом (36) и (37) получаем

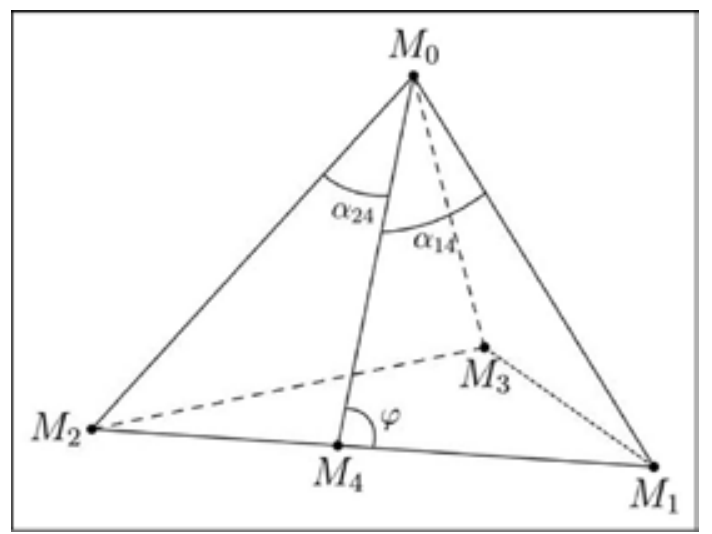

Рис. 3. Схема размещения в пространстве четырех радиоориентиров и фазового центра БПА

Fig. 3. Spatial layout of four radio reference points and the onboard DF antenna phase center 


$$
\ell_{2}=\rho \ell_{1} .
$$

В соответствии с теоремой косинусов для треугольника $\Delta M_{1} M_{0} M_{2}$ (см. рис. 1) получаем соотношение

$$
\ell_{1}^{2}-2 \ell_{1} \ell_{2} \cos \alpha_{12}+\ell_{2}^{2}=d_{12}^{2} .
$$

Из соотношения (39) с учетом (37) и (38) получаем следующую формулу для определения длины ребра $\ell_{1}$ (см. рис. 1):

$$
\ell_{1}=\sqrt{\frac{d_{12}}{1-2 \rho \cos \alpha_{12}+\rho^{2}}} .
$$

С использованием вычисленных по формулам (40) и (38) значений длин боковых ребер соответственно $\ell_{1}$ и $\ell_{2}$ треугольной пирамиды $M_{0} M_{1} M_{2} M_{3}$ (см. рис. 1) для определения длины бокового ребра $\ell_{3}$ треугольной пирамиды $M_{0} M_{1} M_{2} M_{3}$ в системе уравнений (4) из третьего уравнения вычтем второе, в результате чего получаем следующее соотношение:

$$
\ell_{3}=\frac{\ell_{1}^{2}-\ell_{2}^{3}+d_{23}^{2}-d_{13}^{2}}{2\left(\ell_{1} \cos \alpha_{13}-\ell_{2} \cos \alpha_{23}\right)} .
$$

Анализ результатов исследования. Обоснование предложенных алгоритмов однозначного определения координат и угловой ориентации БПА, размещенной на подвижном объекте, по результатам азимутально-угломестного радиопеленгования минимально возможного числа радиоориентиров, равного трем, связано в первую очередь с необходимостью упрощения реализации размещения в пространстве системы радиоориентиров. При определении координат и угловой ориентации БПА, размещенной на подвижном объекте, по результатам азимутальноугломестного радиопеленгования более чем трех радиоориентиров в общем случае точность определения координат и угловой ориентации БПА повышается. Однако привлечение дополнительных радиоориентиров при решении поставленной задачи приводит к необходимости рассмотрения переопределенных несовместных нелинейных систем, что существенно усложняет поиск однозначного их решения относительно искомых параметров.

Покажем, что небольшие погрешности измерений не приводят к несовместности системы уравнений (4). Это обстоятельство оказывается существенным, поскольку поставленную задачу решают в рамках детерминированной модели. Следовательно, можно обойтись без функционала ошибок, построение и изучение свойств которого в случае нелинейных систем является предметом дополнительного исследования. Для этого воспользуемся результатами, изложенными в пункте «Математические особенности определения пространственного положения БПА». На поверхности «закрытого» тора (см. рис. 2) один из плоских углов $\alpha_{12}$, $\alpha_{13}$ или $\alpha_{23}$ при вершине $M_{0}$ треугольной пирамиды $M_{0} M_{1} M_{2} M_{3}$ (см. рис. 1) оказывается постоянным. Если перемещаться по линии пересечения двух таких «закрытых» торов, то два плоских угла при вершине $M_{0}$ треугольной пирамиды $M_{0} M_{1} M_{2} M_{3}$ остаются постоянными, а третий меняется. Следовательно, имеется возможность построения пирамиды $M_{0} M_{1} M_{2} M_{3}$, соответствующей данным измерений плоских углов $\alpha_{12}, \alpha_{13}$ или $\alpha_{23}$ с малыми погрешностями путем поочередного изменения на небольшую величину измеряемых значений всех трех углов $\alpha_{12}, \alpha_{13}$ или $\alpha_{23}$ при вершине $M_{0}$ треугольной пирамиды $M_{0} M_{1} M_{2} M_{3}$. Следовательно, си-

$$
-307-
$$


стема уравнений (4) остается совместной, и для ее решения не требуется строить функционал ошибок.

Когда число радиоориентиров больше трех и никакие три из них не находятся на одной прямой, с учетом полученных результатов исследования может быть предложена следующая процедура однозначного определения координат и угловой ориентации БПА, размещенной на подвижном объекте, по результатам азимутально-угломестного радиопеленгования радиоориентиров: во-первых, выбирют несколько троек радиоориентиров; во-вторых, для каждой выбранной тройки радиоориентиров решают систему уравнений (4) рассмотренными способами; в-третьих, полученные решения согласуют с помощью метода наименьших квадратов или простым усреднением. Построение процедуры «отбраковки» ложных решений, основанной на использовании дополнительной информации при одновременном азимутально-угломестном радиопеленговании более чем трех чисел радиоориентиров, требует дополнительного исследования. Более продуктивным выглядит подход, когда из имеющихся радиоориентиров в каждый момент времени для проведения измерений выбирают тройку радиоориентиров, пространственное положение которой относительно ФЦ БПА обеспечивает наибольшую точность измерений. При этом критерии оптимальности выбора тройки радиоориентиров следует формировать с учетом устойчивости проводимых расчетов.

Для однозначного определения координат и угловой ориентации бортовой пеленгаторной антенны, размещенной на подвижном объекте, по результатам азимутально-угломестного радиопеленгования радиоориентиров предложена четырехэтапная процедура, включающая, вопервых, нахождение совокупности расстояний от фазового центра БПА до радиоориентиров; во-вторых, определение областей пространства, в которых совокупность расстояний от фазового центра БПА до радиоориентиров является однозначной; в-третьих, определение координат фазового центра БПА; в-четвертых, нахождение матрицы вращения и связанных с нею углов Эйлера, определяющих угловую ориентацию в пространстве БПА. Решение задачи нахождения совокупности расстояний от фазового центра БПА до радиоориентиров угломерным методом имеет сложную структуру. Аналитические методы определения совокупности расстояний от фазового центра БПА до радиоориентиров по результатам их азимутально-угломестного радиопеленгования с борта подвижного объекта возможны, но приводят к уравнениям высоких степеней с возможным вырождением порядков уравнений, что означает неустойчивость прямых методов их решения. По-видимому, возможно применение методов регуляризации, но это требует дополнительного математического обоснования. Поэтому предложен устойчивый численный метод решения задачи нахождения совокупности расстояний от фазового центра БПА до радиоориентиров угломерным методом, позволяющий в случае осуществления периодического азимутально-угломестного радиопеленгования с борта подвижного объекта трех радиоориентиров, расположенных в вершинах треугольника, однозначно определять координаты и угловую ориентацию бортовой пеленгаторной антенны, размещенной на подвижном объекте. Предложен вариант размещения в пространстве четырех радиоориентиров, три из них расположены на одной прямой, при котором решение задачи нахождения совокупности расстояний от фазового центра БПА до радиоориентиров на основе использования результатов их азимутально-угломестного радиопеленгования с борта подвижного объекта является единственным и определяется в виде аналитических соотношений. 


\section{Список литературы / References}

[1] Яценков B.С. Основы спутниковой навигащии. Система GPS NAVSTAR и ГЛОНАCC. М.: Горячая линия - Телеком, 2005. 272 c. [Yatsenkov V.S. Basics of satellite navigation. GPS, NAVSTAR and GLONASS Systems. Moscow: Goryachaya liniya - Telekom. 2005. 272 p. (in Russian)].

[2] Тяпкин В.Н., Гарин Е.Н. Методы определения навигационных параметров подвижных средств с использованием спутниковой радионавигационной системы ГЛОНАСС: монография. Красноярск: Сибирский федеральный университет, 2012. 260 с. [Tyapkin V.N., Garin E.N. Methods for determining the navigation parameters of moving objects using the GLONASS satellite radio navigation system: monograph. Krasnoyarsk: SFU. 2012. 260 p. (in Russian)].

[3] Пестряков В.Б. Радионавигаиионные угломерные системы. М.-Л.: Госэнергоиздат, 1955. 304 c. [Pestryakov V.B. Radio navigation angle measuring systems. M.-L.: Gosenergoizdat, 1955. 304 p. (in Russian)].

[4] Перов А.И., Харисов В.Н. ГЛОНАСС. Принципь построения и функиионирования. 4-е, перераб. и доп. изд-е. М.: Радиотехника, 2010. 800 с. [Perov A.I., Kharisov V.N. GLONASS. Principles of construction and operation. Ed. 4th, rev. and add. M.: Radiotekhnika. 2010. 800 p. (in Russian)].

[5] Корнев В.В., Чмутин Н.Ф. Определение пространственной ориентации объекта в среде глобальных радионавигационных спутниковых систем. Ракетно-космическое приборостроение и информационные технологии. 2016. Сборник трудов VIII Всероссийской научно-технической конференции «Актуальные проблемы ракетно-космического приборостроения и информационных технологий» (1-3 июня 2016 г.). Под ред. д.т.н., профессора А.А. Романова. М.: АО PKC, 2016, c. 52-69. [Kornev V.V., Chmutin N.F. Determination of spatial orientation of an object by global radio navigation satellite. Rocket and Space Engineering and Information technologies. Proceedings of VIII all-Russian scientific-technical Conf. "Topical issues of Rocket and Space Engineering and Information technologies". (1-3 June 2016). Edited by Professor Romanov A.A. M.: AO RKS, 2016. 52-69. (in Russian)].

[6] Белавин О.В. Основы радионавигации. Изд. 2-е, перераб. и доп. М.: Сов. радио, 1977. 320 c. [Belavin O.V. The basics of radio navigation. Ed. 2nd rev. M.: Sov. radio, 1977. 320 p. (in Russian)].

[7] Беляевский Л.С., Новиков В.С., Олянюк П.В. Основы радионавигации. М.: Транспорт, 1982. 288 c. [Belyayevskiy L.S., Novikov V.S., Olyanyuk P.V. The basics of radio navigation. M.: Transport, 1982. 288 p. (in Russian)].

[8] Ярлыков М.С. Статистическая теория радионавигаиии. М.: Радио и связь, 1985. 344 с. [Yarlykov M.S. Statistical theory of radio navigation. M.: Radio i svyaz, 1985. 344 p. (in Russian)].

[9] Сосулин Ю.Г. Теоретические основы радиолокаиии и радионавигации. М.: Радио и связь, 1992. 304 с. [Sosulin Yu.G. Theoretical Foundations of Radar and Radio Navigation. М.: Radio i svyaz, 1992. 304 p. (in Russian)].

[10] Бакулев П.А., Сосновский А.А. Радиолокационные и радионавигачионные системы: М.: Радио и связь, 1994. 296 с. [Bakulev P.A. Sosnovskiy A.A. Radar and radio navigation systems. M.: Radio i svyaz, 1994. 296 p. (in Russian)].

[11] Бранец В.Н., Шмыглевский И.П. Введение в теорию бесплатформенных инерциальных навигационных систем. М.: Наука, 1992. 280 с. [Branets V.N.. Shmyglevskiy I.P. Introduction to the theory of strapdown inertial guidance and navigation systems. M.: Nauka, 1992. 280 p. (in Russian)].

$$
-309-
$$


[12] Алешин Б.С., Веремеенко К.К., Черноморский А.И. Ориентаџия и навигаџия подвижных объектов: современные информационные технологии. М.: ФИЗМАТЛИТ, 2006. 424 с. [Aleshin B.S., Veremeyenko K.K., Chernomorskiy A.I. Orientation and navigation of moving objects: modern information technology. M.: FIZMATLIT, 2006. 424 p. (in Russian)].

[13] Виноградов А.Д., Востров А.Ю., Дмитриев И.С. Оценивание пространственной ориентации подвижного объекта по показаниям ортогональных акселерометра и магнитометра. Успехи современной радиоэлектроники, 2016, 7, 35-45 [Vinogradov A.D., Vostrov A.Yu., Dmitriyev I.S. Estimation of the spatial orientation of a moving object according to the readings of an orthogonal accelerometer and magnetometer. Achievements of modern radioelectronics, 2016, 7, 35-45 (in Russian)].

[14] Чаплыгин А.А., Семенов Н.Н., Лукьянчиков В.Д., Медведев А.Б. Использование бортовых навигационных комплексов для решения задач в различных радиотехнических приложениях. Антенны, 2014, 9(208), 40-56 [Chaplygin A.A., Semenov N.N., Lukianchikov V.D., Medvedev A.B. Using on-board navigation systems to solve problems in various radio engineering applications. Antennas, 2014, 9(208), 40-56 (in Russian)].

[15] ГОСТ 22268-76 Геодезия. Термины и определения. М.: Издательство стандартов, 1977. 34 c. [GOST 22268-76 Geodesy. Terms and definitions. M.: Izdatelstvo standartov, 1977. 34 p. (in Russian)].

[16] Виноградов А.Д., Востров А.Ю., Дмитриев И.С. Обобщенная структура радиопеленгатора и основные термины, используемые в теории радиопеленгования. Антенны, 2018, 5(249), 5-20 [Vinogradov A.D.. Vostrov A.Yu.. Dmitriyev I.S. Generalized structure of the direction finder and basic terms used in the theory of radio direction finding. Antennas, 2018, 5(249), 5-20 (in Russian)].

[17] Ишлинский А.Ю. Ориентация, гироскопы и инерциальная навигащия. М.: Наука, 1976. 622 c. [Ishlinskiy A.Yu. Orientation, gyroscopes and inertial navigation. M.: Nauka, 1976. 622 p. (in Russian)].

[18] ГОСТ Р 51794-2008. Глобальные навигационные спутниковые системы. Системы координат. Методы преобразования координат определяемых точек. М.: Стандартинформ, 2009. 16 c. [GOST R 51794-2008. Global navigation satellite systems. Coordinate systems. Methods for transforming coordinates of defined points. M.: Standartinform, 2009. 16 p. (in Russian)].

[19] ГОСТ 20058-80 Динамика летательных аппаратов в атмосфере. Термины, определения и обозначения. М.: Издательство стандартов, 1981. 56 с. [GOST 20058-80 The dynamics of aircraft in the atmosphere. Terms, definitions and designations. M.: Izdatelstvo standartov, 1981. 56 p. (in Russian)].

[20] Бахвалов Н.С., Жидков Н.П., Кобельков Г.Г. Численные методы. Изд-е 8. М.: Лаборатория Базовых Знаний, 2000. 636 c. [Bakhvalov N.S., Zhidkov N.P., Kobelkov G.G. Numerical methods. Ed. 8th. M.: Laboratoriya Bazovykh Znaniy, 2000. 636 p. (in Russian)]. 